Rätsel der Schöpfung

\title{
Das wundersame Spektrum der Gliflozine
}

Die molekulare Medizin hat das Ziel, die Prozesse in und um organische Zellen im Detail zu verstehen - theologisch ausgedrückt: die Schöpfung zu ergründen. Mit diesem detaillierten Wissen - so die Hoffnung der Molekular-Forscher - sollen Erkrankungen entschlüsselt und maßgeschneiderte Therapien entwickelt werden. Im Bereich der Diabetologie hat man diesen Weg bei der Charakterisierung des PPAR-gamma Rezeptors beschritten. Es konnte gezeigt werden, dass dieser bei Insulinresistenz eine wichtige Rolle spielt. Anschließend wurden mit den Glitazonen Medikamente geschaffen, die beim Menschen die Insulinsensitivität verbessern und zu einem Absinken der Blutzuckerwerte führen. In klinischen Studien sind dann nicht unerhebliche Komplikationen zu Tage getreten, da PPAR-gamma Rezeptoren auch in anderen Zellen vorhanden sind und bei Aktivierung ungünstige Effekte auslösen können.

\section{Von der Natur lernen}

Ein anderer Weg, der in der medizinischen Forschung über viele Jahrzehnte beschritten wurde, ist die Einzelbeobachtung, also „von der Natur zu lernen“. Dieser klassische Weg wurde zuletzt bei der Entwicklung der SGLT-2-Inhibitoren verfolgt. Grundlage war die klinische Beobachtung, dass es Menschen ohne Diabetes mellitus gibt, die Glukose im Urin ausscheiden. In molekularen Analysen konnte die Ursache dieser familiär auftretenden Glukosurie in Mutationen im SGLT-2-Gen gefunden werden. Je nach Lage der Mutation ist die Funktion dieses für die Glukoserückresorption in der Niere wichtigen Proteins mehr oder weniger beeinträchtigt. Dieses Wissen hat zur Entwicklung der Gliflozine geführt, die diesen Rezeptor blockieren.

Obwohl hier ein Mechanismus von der Natur abgeschaut wurde, waren die Bedenken in der Diabetologie von Anfang an sehr groß. Dabei spielte vermutlich eine wichtige Rolle, dass die Diabetologie über Jahrzehnte die These vertreten hatte, dass Glukose im Urin schlecht ist - mit der SGLT-2 Inhibition sollte dies nun gut sein? Für den Einsatz von SGLT-2-Inhibitoren bei Typ-2-Diabetes wurden schwere Nebenwirkungen in Form von Harnwegsinfekten und sogar Nierenversagen vorhergesagt, obwohl die familiäre Glukosurie ohne Symptome auftritt. Die meisten klinischen Studien mit Gliflozinen zeigten aber, dass die Rate an Harnwegsinfekten nicht erhöht ist, auch wenn man den deutlichen Anstieg der Genitalinfektionen nicht verharmlosen sollte. Die Sorgen hinsichtlich des Nierenversagens wurden durch die aktuelle Auswertung der EMPA-REG-OUTCOME-Studie sogar ins Gegenteil gewendet, denn für das Präparat Empagliflozin wurde bei Personen mit eingeschränkter Niereninsuffizienz sogar ein protektiver Effekt nachgewiesen (s.

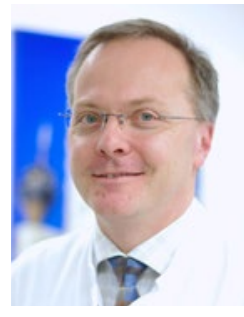

Prof. Dr. med. Stephan Martin

Chefarzt für Diabetologie und Direktor des

Verbund der Katholischen Kliniken

Düsseldorf (VKKD), Westdeutschen Diabetes-

und Gesundheitszentrum (WDGZ)

Hohensandweg 37, 40591 Düsseldorf

Stephan.Martin@vkkd-kliniken.de

Rubrik „aktuell“ S. 43 im Heft). Diese Analysen waren quasi noch ein „ad on“ auf die bereits überraschenden Ergebnisse der Hauptstudie, die eine deutliche Reduktion der kardiovaskulären und der Gesamtsterblichkeit bei Personen mit Typ-2-Diabetes und einem hohen kardiovaskulären Risiko gezeigt hatten.

\section{Das eine nutzt, das andere schadet der Niere?}

Die Datenlage wird aber noch komplizierter. Parallel zu diesen Ergebnissen wurde von der FDA inzwischen für zwei andere SGLT-2-Inhibitoren, Cana- und Dapagliflozin, ein „warning letter" bei Personen mit Niereninsuffizienz herausgegeben [1]. Bedeutet dies, dass wir hier Substanz- und keine Klasseneffekte haben? Zum Leidwesen der molekularen Medizin kann man sich die überraschenden Befunde der EMPA-REG-OUTCOME-Studie überhaupt nicht erklären, sodass nun in diversen In-vitro- und In-vivo-Systemen begonnen wird, dem auf den Grund zu gehen.

Trotz der interessanten Ergebnisse scheint die Diabetologie von der Wirkstoffklasse nicht vollends überzeugt zu sein. Ende Juni hatten die FDA-Experten eingeladen, um die Frage zu klären, ob die Ergebnisse der EMPA-REG-OUTCOME-Studie „substanzielle Evidenz“ liefern, dass Empagliflozin die Zusatzindikation „zur Reduktion der kardiovaskulären Mortalität bei Risikopatienten mit Typ-2-Diabetes“ erhält. Anscheinend nur durch die Stimmen der Kardiologen - und nicht durch die der Endokrinologen und Diabetologen - gab es eine hauchdünne Mehrheit dafür. Die Befunde der EMPA-REG-OUTCOME-Studie zeigen einmal mehr, dass der menschliche Organismus komplizierter ist, als wir es trotz vieler Erkenntnisse der molekularen Forschung wahr haben wollen. Wir werden die Schöpfung wohl nie komplett ergründen!

Literatur:

1. http://www.fda.gov/Drugs/DrugSafety/ucm505860.htm 